\title{
EMDR Treatment in People with Mild ID and PTSD: 4 Cases
}

\author{
L. Mevissen $\cdot$ R. Lievegoed $\cdot$ A. de Jongh
}

Published online: 6 August 2010

(C) The Author(s) 2010. This article is published with open access at Springerlink.com

\begin{abstract}
Although there is evidence to suggest that people with intellectual disabilities (ID) are likely to be more susceptible to the development of posttraumatic stress disorder (PTSD) than persons in the general population, until now only eight case reports on the treatment of people with ID suffering from PTSD symptoms have been published. In an effort to enrich the literature on this subject, the aim was to investigate the applicability of an evidence-based treatment for PTSD (i.e., EMDR) in four people with mild ID, suffering from PTSD following various kinds of trauma. In all cases PTSD symptoms decreased and the gains were maintained at 3 months to 2.5 year follow-up. In addition, depressive symptoms and physical complaints subsided, and social and adaptive skills improved. It is concluded that clients' improvements converge to suggest the applicability of EMDR in people with mild ID. Difficulties involved in arriving at an accurate PTSD diagnosis in ID clients are discussed.
\end{abstract}

Keywords Intellectual disability - EMDR - Posttraumatic stress disorder - Trauma . Treatment

L. Mevissen ( $\square)$

Child and Adolescent Psychiatry (KJP) Drenthe/Overijssel, Accare, Centre for Child and Adolescent Psychiatry, Diepenveenseweg 169, 7413 AP Deventer, The Netherlands

e-mail: 1.mevissen@accare.nl

R. Lievegoed

Zonnehuizen, Centre for Mental Health in Intellectual Disability, Zeist, The Netherlands

e-mail: r.lievegoed@planet.nl

A. de Jongh

Department of Behavioural Sciences, Academic Centre for Dentistry Amsterdam (ACTA),

Universiteit van Amsterdam and Vrije Universiteit, Amsterdam, The Netherlands

e-mail: a.d.jongh@acta.nl 


\section{Introduction}

Certain events are so overwhelming that they clearly leave behind deep impressions. People who have experienced, witnessed, or been confronted with such an event may develop a pathological stress response syndrome, such as posttraumatic stress disorder (PTSD) [1]. To meet criteria for PTSD, an individual must have been exposed to a traumatic stressor, followed by a subjective response of fear, helplessness or horror. The principal clinical feature of PTSD is the painful re-experiencing of the traumatic incident in the form of recurrent and distressing recollections of the event, including intrusive images, nightmares and flashbacks [1]. Other hallmark symptoms of PTSD are avoidant reactions and symptoms of arousal [1]. In the general population PTSD prevalence rates vary between 5 and $10 \%$ [2], while a lower developmental level appears to coincide with a higher PTSD risk and more serious PTSD symptoms [3, 4].

Although their cognitive impairments may make individuals with ID more susceptible to the development of PTSD, those who work with this population often lack this awareness [5]. Furthermore, it is unclear to what extent individuals with ID suffer from the same PTSD symptoms as people without ID. To this end, recommendations for adapted PTSD symptom description in people with ID have been proposed [6]. Moreover, reliable and valid instruments for assessing PTSD in this population are lacking, there are no PTSD prevalence data, and there are no evidence-based treatment methods available for people with ID who suffer from PTSD [5].

Regarding PTSD treatment with ID clients, several evidence-based treatment methods have been utilized. Among them, trauma-focused cognitive behavioral therapy (CBT) and Eye Movement Desensitization and Reprocessing (EMDR) have the strongest empirical support [7-10]. However, to date only eight case reports of PTSD treatment in individuals with ID have been found in the literature $[5,11,12]$. In seven of these case reports CBT (Table 1) or EMDR (Table 2) was employed. In most studies however, valuable information, including follow-up data, was lacking.

In an effort to enrich the literature regarding PTSD treatment in people with ID, this study explores the applicability and efficacy of EMDR in a sample of four people with mild ID, children as well as adults, who experienced various types of negative life events. The findings are discussed with consideration for the difficulties in establishing a PTSD diagnosis in people with ID [5].

\section{Method}

Participants

Subjects were four clients with mild ID in the Netherlands who were referred for outpatient treatment by a mental health professional, a physician or a psychiatrist. All clients suffered from PTSD-like symptoms, according to DSM-IV TR [1] and DM-ID [6] criteria. Symptoms and/or behaviors of the clients were supposed to be related to one or more overwhelming negative life events. Except for long-standing medication (clients \#2 and \#3), EMDR was the sole treatment administered during the course of the study. 


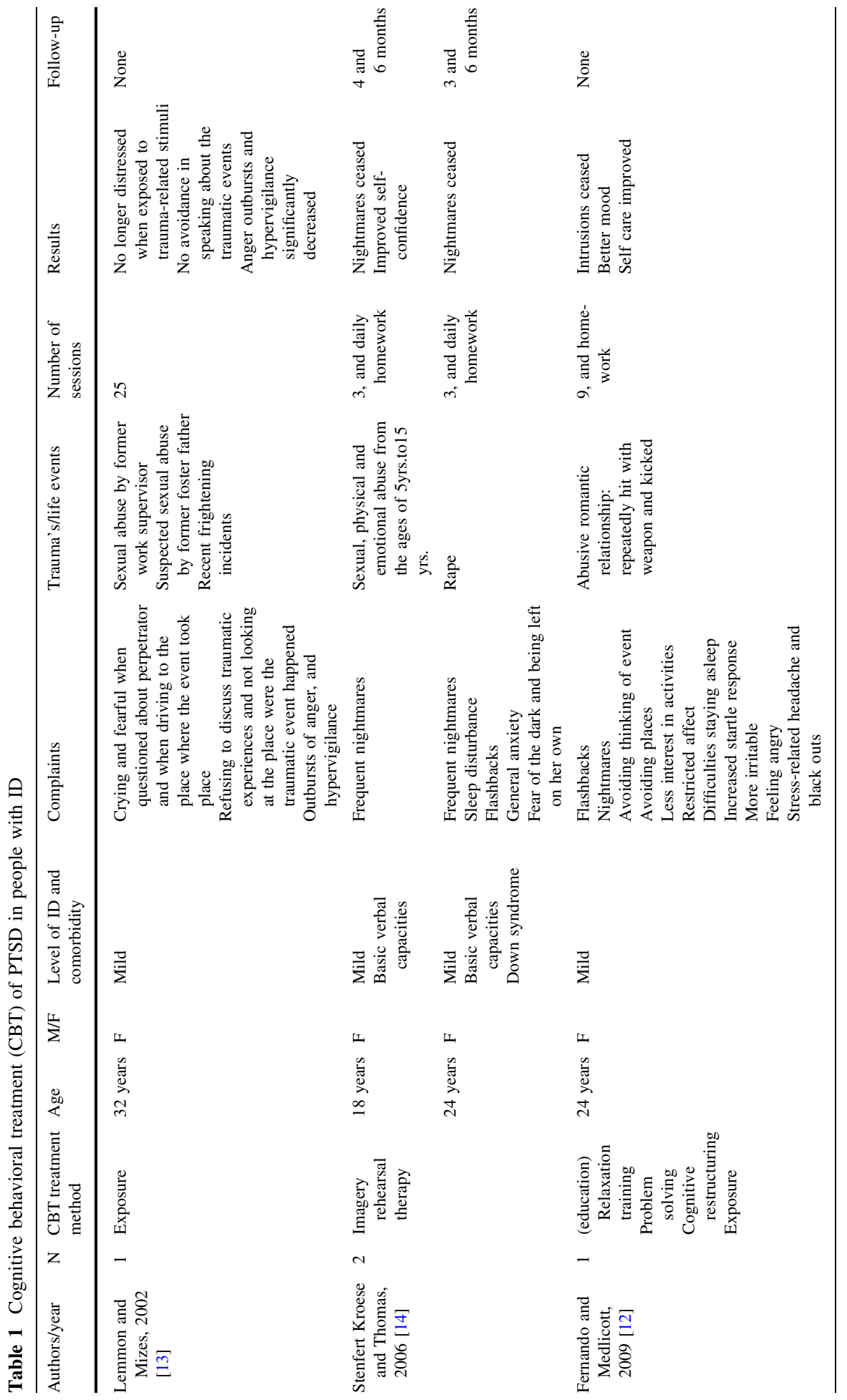




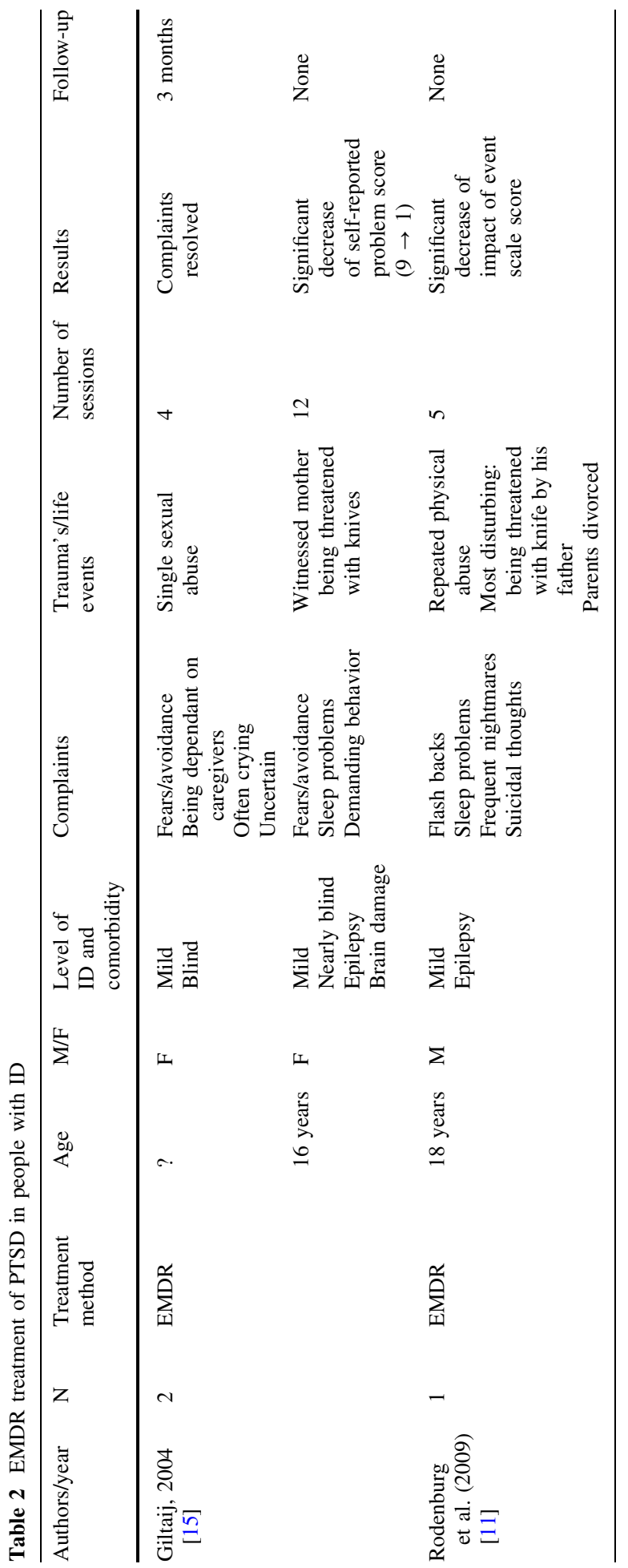


Procedure

The first author determined appropriateness of each participant for treatment based on client's records and by means of a clinical interview with the client and caregiver. Information about the treatment and related procedures were explained in detail to the caregivers, who were also given written information about the treatment to be read at home.

All treatments were administered by the first author, who is a clinical psychologist and a registered EMDR practitioner with more than 30 years experience with ID clients. Treatment was provided in weekly sessions of $60 \mathrm{~min}$ each of which the exact number of sessions was based on their clients' individual needs. All sessions were videotaped with written consent of the client or caretaker. During treatment all clients, except one (client \#3), were accompanied by a parent or professional caregiver in order to provide a sense of safety, to overcome communication disabilities, to facilitate an integration of the therapeutic process and daily life, and/or to function as a co-therapist. In each session clients, parents or caregivers were asked to report observed changes in daily life functioning, with specific attention to complaint-related behaviors.

\section{Treatment}

Eye Movement Desensitisation and Reprocessing (EMDR) is a psychotherapeutic approach, developed to resolve symptoms resulting from disturbing and unresolved life experiences [16]. Eleven randomized controlled trials have evaluated the effects of the eye movements in relation to memory and demonstrated a variety of effects including emotional dearousal, decrease in imagery vividness and episodic retrieval [8]. EMDR is based on a theoretical, information processing model, which posits that the symptoms are likely to disappear when the memories are fully processed and integrated [16, 17] It requires the client to attend a bilateral "dual attention" stimulus (typically the therapist's fingers moving back and forth in front of client's face; sometimes audio tones or hand taps are used) while concentrating on the trauma memory [18].

Briefly, EMDR treatment consists of (1) Taking history and planning treatment (2) an explanation of and preparation for memory processing, (3) preparation of the target memory, in which the client is asked to focus on the worst moment of the memory in a multi-modal manner, including image, thought, emotion and physical sensation, with concomitant measurements and (4) active reprocessing of the memory. In the desensitization phase, the therapist asks the client to hold the target image in mind while concentrating on the bilateral stimulation (BLS) for about $30 \mathrm{~s}$. The client reports briefly what comes up, and is guided by the therapist to refocus attention along with the stimulus according to standardized procedures. During subsequent 'sets' associations that arise may become the focus of attention. Processing continues until the client reports no remaining distress related to the memory. The client is then (5) guided to embrace a relevant positive belief regarding the event and to (6) identify and process any residual disturbing body sensations. The therapist then facilitates (7) a positive closure to the session, which is followed by (8) a re-evaluation during the subsequent session, in which the client comments on previously processed targets as a basis for guiding further intervention.

The EMDR procedure in the present study is the Dutch translation [19] of Shapiro's [18] protocol, and has been adapted to the level of clients' cognitive and emotional functioning as suggested by Tinker and Wilson [20], Lovett [21], Greenwald [22], De Roos and Beer [23] and Seubert [24]. In general a short explanation of the treatment in terms of 
"exercises to get rid of bad feelings about events" or "to help you fix the problem that brought you here" has been found to be sufficient. Parents or caregivers play a significant role in motivating the client, either as an information source for the therapist, as a cotherapist if the developmental level is significantly impaired, and/or as a support in reinforcing positive behaviors. In general, in contrast to the usual utilization, the therapist's attitude is more directive without taking over, and language is simplified. Visual cues are used instead of abstract language, for example using facial images to represent feelings, or a drawing might represent the target memory if the person is not able to mentally visualize it. Physical gestures help communication. By spreading hands or arms apart or bringing them closer, the client can indicate the amount of disturbance related to the target image. Parts of the protocol that appear to be too difficult are eliminated.

When the person is unable to perform eye movements, other forms of bilateral stimulation are used, primarily bilateral sound and tactile stimulation. From a developmental level of \pm 3 years downward the Story Telling Method [21] is employed, with parents or caregivers narrating the story. The story will have a positive start, recognizable by the client. The next part of the story concentrates on the traumatic events, including distressing details the person might have seen, heard, felt, thought or smelled. The way in which the client had responded to the symptoms that emerged after the event was included. Finally, a recognizable positive ending is formulated as well as positive client self-beliefs. During the entire story, bilateral stimulation is applied. The story is repeated until the disturbance related to the target image has disappeared (SUD $=0$ ). Between the story narrations, an enjoyable game can be suggested to relax the client and to emphasize safety and empowerment in present time, in contrast to the temporary disturbance from the traumatic past. In the beginning of each session a positive self-belief may be installed, gleaned from some recent positive experience.

The primary treatment goal for all clients was to eliminate PTSD-like symptoms.

In the following case descriptions client's level of functioning is represented as noted in the client's records.

\section{Results (see also Table 3)}

\section{Client 1}

John is a 32-year old man with mild ID (WISC-R: Dutch version of the Wechsler intelligence scale for children-revised [25, 26]; Total IQ 69, Verbal IQ 70, Performance IQ 69). He lives in a supervised apartment in a large city and works in a sheltered workshop. 5 years ago he had a severe scooter accident. Since then John has been extremely frightened whenever he sees a motor vehicle or scooter accident on television. Each year he feels badly on the anniversary of the accident. John's stress impacts greatly on his intestines, requiring approximately 20 daily visits to the bathroom. At work he is not able to tolerate criticism and his pace is slow. Riding had always been his favourite hobby, but since the accident he has avoided horses. John does not remember anything about the accident itself, since he lapsed into a coma. He becomes agitated at the thought of the man who, after he woke up from coma, accused him of jumping the traffic lights, something he said never happened before. In his files other negative life events were discovered. His parents had divorced when he was at the age of 12 and when 22 years old he was accused of sexual abuse and was treated for that. 
The scooter accident was treated in the first session. He described the target image, which he could mentally visualize, as "the car en me lying on the ground". He was thinking "I am in danger", he felt anxiety and rated the distress as 10 on a $0-10$ scale, located in his stomach. Bilateral stimulation was applied by using an electronic auditory device. After fully reprocessing this traumatic memory, a future template was installed: "I am riding again and feel relaxed". During the second session John revealed that he was suffering from outbursts of anger and he reported that there was another overwhelming event that bothered him much of the time. His girlfriend broke off their engagement after having been together for 4 years. Enraged, he hit her and now considered himself a criminal as a result. In the target image he saw himself hitting his girlfriend, and judged himself an "abuser". By way of contrast, he wanted to perceive himself as "being good with girls". The latter preferred belief did not feel very true (he rated this a four on a 1-7 scale with one feeling not true at all and seven feeling completely true). He felt angry, with a distress of seven on a $0-10$ scale located in the upper part of his body. The event was reprocessed during that second session.

At a 4 month follow-up, John was no longer upset when experiencing scooter accidents. He was able to take the same route to work as he had before the accident (John had not mentioned earlier that he had been avoiding this route since the accident). The anniversary of the accident passed without his being triggered. He was very proud that he had started riding again; a bit stressful at first, but manageable. Outbursts of anger as well as physical complaints had decreased. He was doing well at work and he noticed he was working more efficiently. The only problem remaining was the fact that he was still single. He asked his care giver to help him learn how to create new relationships.

Recently, 28 months later, John's now former caregiver visited him on his birthday. John was doing very well. The positive effects of therapy had lasted, and John had succeeded in building a new, stable relationship, which he was still enjoying after 2 years.

\section{Client 2}

Mitchell is an 11-year old boy with mild ID (Total IQ 61, Verbal IQ 72, Performance IQ 55). He has been diagnosed with autism spectrum disorder, specified as multiple complex developmental disorder (MCDD; [27]). Prominent are fears, compulsive behavior, obsessions (e.g., fires), hearing voices and difficulties distinguishing reality from fantasy. Pipamperon, a mild antipsychotic, had been prescribed for a period of 4 years. Play therapy had ended a few months before an EMDR referral. At age 7 Mitchell had been removed from the home because his parents were not able to control his behaviors. After two temporary placements he was eventually put in a group home and a special needs school at the age of 8 and is functioning quite well in both. Due to considerable fear, he avoids sleeping at home where his mother lives together with her third partner, Mitchell's brother and little sister. Because he hears voices urging him to act out aggressively, Mitchell's visits to his mother's home are accompanied by outbursts of anger and fights with his brother. The visits are consequently limited and require the presence of a caregiver.

Mitchell was able to draw and successfully process the following overwhelming events that still bothered him: (1) as a little boy, voices forced him to throw things. He threw a vase and his little brother started crying: (2) a car fire in front of his family home: (3) a quarrel between his mother and her friend; (4) a "nasty man" was at the house ordering him to speak to his mother; the police were called, but never arrived. Mitchell preferred the combination of both auditory (headphones) and tactile (buzzers) stimulation. The choice of the sequence of target memories was left to Mitchell. He decided to begin with the target 
image of "the nasty man". In this memory, the target image of seeing the nasty man standing in the door, was accompanied by the negative belief "I am in danger", a feeling of anger with distress rated 9 on a $0-10$ scale, and a ticklish feeling in his belly. His second choice centered around the image of seeing his mother coming back from a first aid station, with his negative thought "I can't do anything". He felt angry, sad and anxious simultaneously, and again reported a distress of nine with the same ticklish feeling in his belly. In the third memory he targeted the image of "the car, completely black with broken windows". His corresponding, presently held, negative belief was "I can't do anything" and he pointed to his right forearm as the location of an anxious feeling which he rated at a maximum level of ten. The fourth session targeted an intense fight between his mother and his first stepfather, which resulted in his stepfather packing his bags, and leaving the house for good. He brought up this event in light of a recent nightmare. Mitchell described the corresponding target image as follows: "My stepfather is standing in front of my mother and shaking his hands at her. I see my self close to them, and I'm afraid of my stepfather's angry outburst". After these memories had been successfully processed and resolved, Mitchell chose to address, the one centering around the voices in his head that had caused him to throw the vase. To his great surprise, while preparing this target memory, he felt no distress at all, "without using these!", he exclaimed, pointing to the buzzers and the headphones on the table.

After the five sessions needed to process these memories, the voices had disappeared and the short visits at his parental home went smoothly. Mitchell became calmer, and his compulsive behaviors decreased. The visits were extended and 6 weeks later he had slept at home three times without any reported difficulties. His problematic behaviors completely disappeared, despite the tumultuous atmosphere of his group home and his mother's partner leaving her. During the next 6 weeks a systematic phasing out of his medication was completed, and the diagnosis of autism spectrum disorder no longer applied. At another 6 week follow-up, results maintained.

\section{Client 3}

Mary is a 53-year old woman with moderate to mild ID (WISC-R: Dutch version of the Wechsler intelligence scale for children-revised [25, 26]; Verbal IQ 49; SRZ-P: Dutch social adaptive behaviour scale for individuals with a higher level of intellectual disability (Total score 7 [28]). Together with her partner she lives in a supervised apartment and works in a sheltered workshop for a few hours every week.

About 17 years ago she witnessed her brother-in-law choking her father. Since that event Mary has suffered from panic attacks with increasing intensity and frequency. Given an impaired heart valve, and not wanting to take chances when these events occur, the panic attacks often result in hospitalization. Mary gets angry easily and persistently. She has taken an SSRI for a year and a half to reduce her anxiety. Mary reports suffering from nightmares, states that "it's busy in my head", and experiences a ringing sensation in her ears. She often hears her brother-in-law's voice, saying "I'll catch you!". Additionally she avoids a street where she had seen a well.

During history taking a list of other negative experiences was compiled, including the following: at school her notebook was torn apart by a teacher, which resulted in her detesting school; frequent quarrelling at her parent's home; witnessing her brother and brother-in-law beating mother; witnessing her father struggling to breathe and then dying; two divorces; her mother's death 9 years ago; an emergency hospital admission 5 years 
earlier, due to pulmonary embolism and heart valve problems, which in turn led to a number of surgeries.

Mary was driven to her therapy sessions by a trusted volunteer, since her caregiver was not able to accompany her. Information exchange with the caregiver before and after each session was facilitated via e-mail. Since Mary had complained about the impact of the fight between her father and brother-in-law, this memory was targeted first. Headphones were used for auditory bilateral stimulation. In choosing the image of the worst moment of the memory, Mary saw herself witnessing her brother-in-law choking her father. As she held this image, the relevant negative thought was "I can't do anything", which was accompanied by a fear that she could identify in her larynx. It took five sessions to process this trauma. Within a couple of weeks, her care giver reported improvements in Mary's functioning, as she appeared more cheerful and was free of panic attacks. According to Mary, it felt better inside her head. On one particular day after the fourth session of working on this trauma, Mary was brought to the hospital. Although nothing appeared to be wrong, the feeling of tightness in her chest had been most distressing, she reported later on. After the traumatic memory was completely processed, her thinking was more peaceful, she could sleep well, and the panic attacks once again disappeared. The most distressing memory left was the image of her brother, punching her mother in the stomach. It took three sessions to process this memory. Throughout the time of this processing Mary's functioning in her daily life continued to improve. At times there was the feeling of tightness of her chest but it did not overwhelm her, nor did it lead to a panic attack. In the last three sessions, the memories of her father's death and of her own heart surgery were processed, leaving no more distressing memories after a total of 13 reprocessing sessions.

At a 6 month follow-up, panic attacks continued to be absent. Mary slept well and felt cheerful. Her anger outbursts had disappeared, social relationships had improved and she was willing to become involved in new activities. She was even able to walk past the well she had feared. In retrospect, it seems evident that this well had triggered the memory of seeing her father's coffin being lowered into the grave. Mary was admitted to the hospital at one point for a week, due to actual cardiac conditions. Noticeably calm, she cooperated with and underwent the required medical treatment.

Seven months after the first follow-up her caregiver reported that Mary still was doing very well. She remained remarkably calm and cheerful. Voices and panic attacks remained absent. Now and then medical problems occurred, which she dealt with appropriately. The phasing out of her medication had been advised, but caregivers forgot this recommendation, leaving her medication regimen at present unchanged.

\section{Client 4}

Eve is a 7 year-old girl with mild to borderline ID (Total IQ 71, Verbal IQ 82, Performance IQ 64). At the age of six she was diagnosed with childhood disorder NOS with concomitant diagnosis of obsessive compulsive disorder. Recently she was referred for further specialized assessment which resulted in the diagnosis autism spectrum disorder. Eve suffers from anxiety, compulsive behaviors, outbursts of anger, and frequent mood changes, but most of all from disturbing thoughts about illness, death and knives. Life history was gathered with the help of her parents. No painful memories were identified at that time. Consequently, the diagnosis of autism spectrum disorder was changed to the more specific diagnosis of multiple complex developmental disorder (MCDD; [27]). Her parents were trained at home on how to deal with Eve's autism in daily life. A psychiatrist educated them regarding the possibility of medication. Simultaneously, Eve was offered an individual 
training aimed at teaching her to use relaxation techniques to deal with disturbing thoughts. After three such sessions, her symptoms significantly increased after hearing of the death of Michel Jackson. While analyzing that situation, both parents realized that in the past 3 years several overwhelming events had taken place. First of all, Eve experienced a dead body for the first time when a dear family member suddenly died. Secondly, her father's best friend had been missing for several days and was finally found with knife stabs in his body (Eve had not actually seen the knife wounds, but heard the frightening stories concerning that what had happened). Then, another close family member died after a short illness. Finally she saw pictures of her classmate with a life threatening illness, lying in a hospital bed, surrounded by an array of medical equipment.

Given this new information, EMDR treatment was offered in place of the relaxation training. Eve's poor communication skills and her sudden reluctance to follow instructions became obstacles to the use of the standard protocol. Consequently, the story telling method was used. For the BLS, she held buzzers in her hands. Following a preparation session with her parents, it took three sessions to process the traumatic memories. Her disturbing thoughts disappeared, and at a 7 week follow-up, the effects had lasted. Moreover, she exhibited a positive mood more often, her outbursts of anger had significantly decreased, and, in general, she was more relaxed. Unexpectedly after this progress, Eve's parents called again for help, relating that Eve had become anxious while watching television or a DVD. Upon examining the situation, it became clear that it was not an anxiety, but a reactive stress response, since Eve had not been educated as to what movies where good for her to see. With support and care at home, Eve's parents turned "watching films" into a predictably positive activity for Eve. The stress disappeared, and at a 3 month follow up treatment effects had lasted. The use of medication was ruled out. A psychiatric consultation was arranged, and Eve was ultimately diagnosed with Autistic Disorder.

\section{Discussion and Conclusions}

The results of the four cases suggest that EMDR can be used as a treatment for people with mild ID as the application of the procedures resulted in a clear reprocessing of the memories related to the traumatic events. Following treatment, none of the four clients fulfilled the diagnostic criteria of PTSD according to the DSM-IV-TR [1] and DM-ID [6]. Despite their intellectual disabilities, it was possible to adapt instructions to clients' cognitive and emotional level. For example, with clients \#1, \#2 and \#3 the protocol for children was used and matched to their cognitive level of functioning. These clients appeared to be able to put images, thoughts, feelings and physical sensations into words. Despite a cognitive level of mild ID, client \#4 required story telling, due to the subject's severe communication impairments, a prominent feature of her autistic disorder [29].

With regard to treatment efficacy, the decrease of PTSD-like symptoms following a relatively small number of EMDR sessions is in line with the previously published findings of the three EMDR case reports of PTSD treatment in subjects with mild ID [10, 15] (see also Table 2). Additionally, the findings of this study demonstrate that in all four cases PTSD-like symptoms that had been present for 3 to even 17 years, dissipated after 2 to 13 EMDR treatment sessions. Treatment effects remained at the 3 month and the 2.5 year follow-up. Moreover, depressive symptoms (clients \#3 and \#4) and physical complaints (client \#1 and \#3) decreased, developmental growth increased, and social and adaptive skills improved (\#1 and \#3). The observation that positive growth factors occurred 
subsequent to treatment has important implications for this population and should be evaluated in future research.

The complete elimination of the symptoms post-treatment that had caused the caretakers to seek treatment for the clients also sheds light on the need to evaluate ID patients for distressing life events that may be foundation pathology whether or not they are Criterion A events, or if the symptoms raise to full diagnostic criteria for full PTSD [18, 30]. Specifically, the complete treatment effects were derived after processing memories that might be considered "life-threatening", and others that distressed the client such as quarrels, and unfortunate acts by teachers or other authority figures. Further, the present findings should be considered in light of the difficulty in establishing a proper PTSD diagnosis in people with ID, as well as the lack of data regarding this population's treatment responsiveness [5]. Therefore, feedback for the present study was gathered by interviewing parents and caregivers according to DSM-IV-TR [1] and DM-ID criteria [6]. It emerged that all patients had been exposed to more than one overwhelming event, and at least one of the events for each client seemed to meet PTSD criterion A1 for trauma (DSMIV-TR [1]). These criteria include experiencing threat to physical integrity of self (client $\# 3$ ), experiencing the threat of death or personal injury (client \#1), witnessing an actual death (client \# 3), or the threat of death or serious injury to others (client \#2, \#3 and \#4). The second PTSD criterion (A2), DSM-IV-TR [1] includes a response involving intense fear, helplessness or horror. Of the four cases, only clients \#2 and \#3 were able to report emotional awareness while experiencing or witnessing the overwhelming event. This seems to be the core problem with diagnosing people with ID with PTSD, as they often are unable to communicate feelings of helplessness or horror, particularly verbally. Clients with autism, in particular, tend to express their emotions in atypical ways. Consequently, the emotional impact of an event might be concealed even from trusted persons (client \#4). Another complicating factor is that, in comparison with the general population, differences may occur in the expression of symptoms, and in the interpretation of distressing experiences [5]. Based on patients' complaints (see Table 3), re-experiencing of trauma, PTSD criterion B (DSM-IV-TR [1]), appears to be the most recognizable symptom in all four subjects: nightmares, hearing voices (client \#3), being frightened by trauma-related stimuli (client \#1), voices (clients \#2 and \#3), fantasies (client \#2), and disturbing thoughts (client \#4). The difficulty in diagnosing PTSD in clients with ID is perhaps best illustrated when considering PTSD criterion C (DSM-IV-TR [1]), which lists seven signs of avoidance, at least three of which have to be present. This criterion seems hardly applicable to clients with mild ID, because it relies strongly on verbal, subjective reporting. Consequently, with respect to this criterion, the DM-ID [6] places great significance on reports from parents and caregivers regarding signs of "non-compliance" and self-isolation. It is important to note that it was after treatment that the primary caregivers of all clients in this study, except for the one with autism, reported remarkably positive changes in terms of "taking up activities", "improving independence" and "learning new skills". Thus, only with hindsight were the symptoms of avoidance noted. With regard to the client with autism (\#4), it was difficult to notice symptoms of avoidance at all. After treatment however, parents reported that activities were taken up without aggression. Concerning criterion D, the situation is different. In three of four patients (clients \#1, \#2, and \#3) PTSD criterion D (DSM-IV-TR [1]) appeared to be recognizable when considering the presenting complaints at the time of referral. Subject \#4 reported only one of two required symptoms of arousal (i.e. anger outbursts). It is not clear whether other symptoms were really absent or just had not been noted by the caregivers. Remarkably, excessive anger was mentioned in all four patients. In subjects \#2 and \#4, traumatic re-experiencing was misinterpreted as a psychotic 


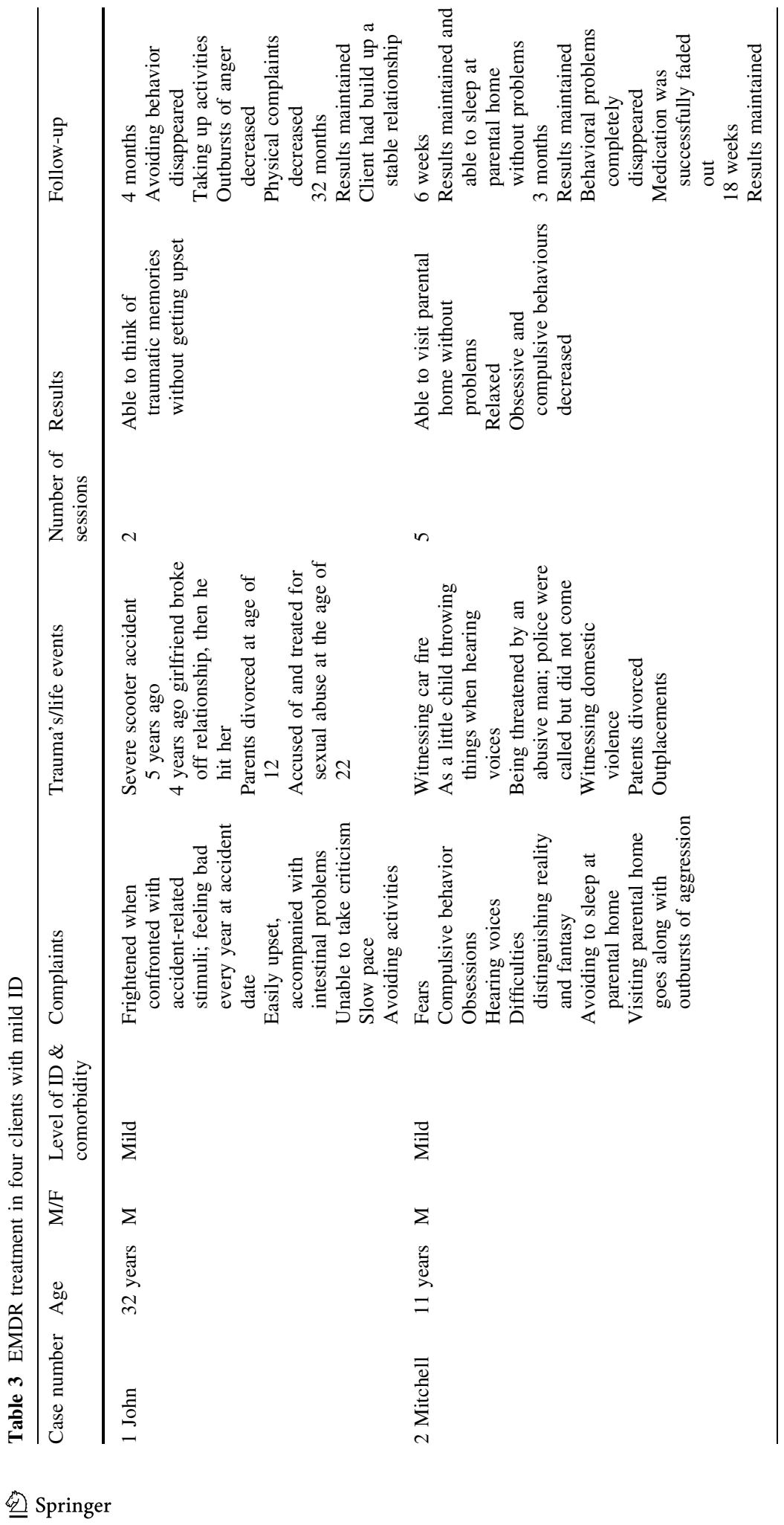




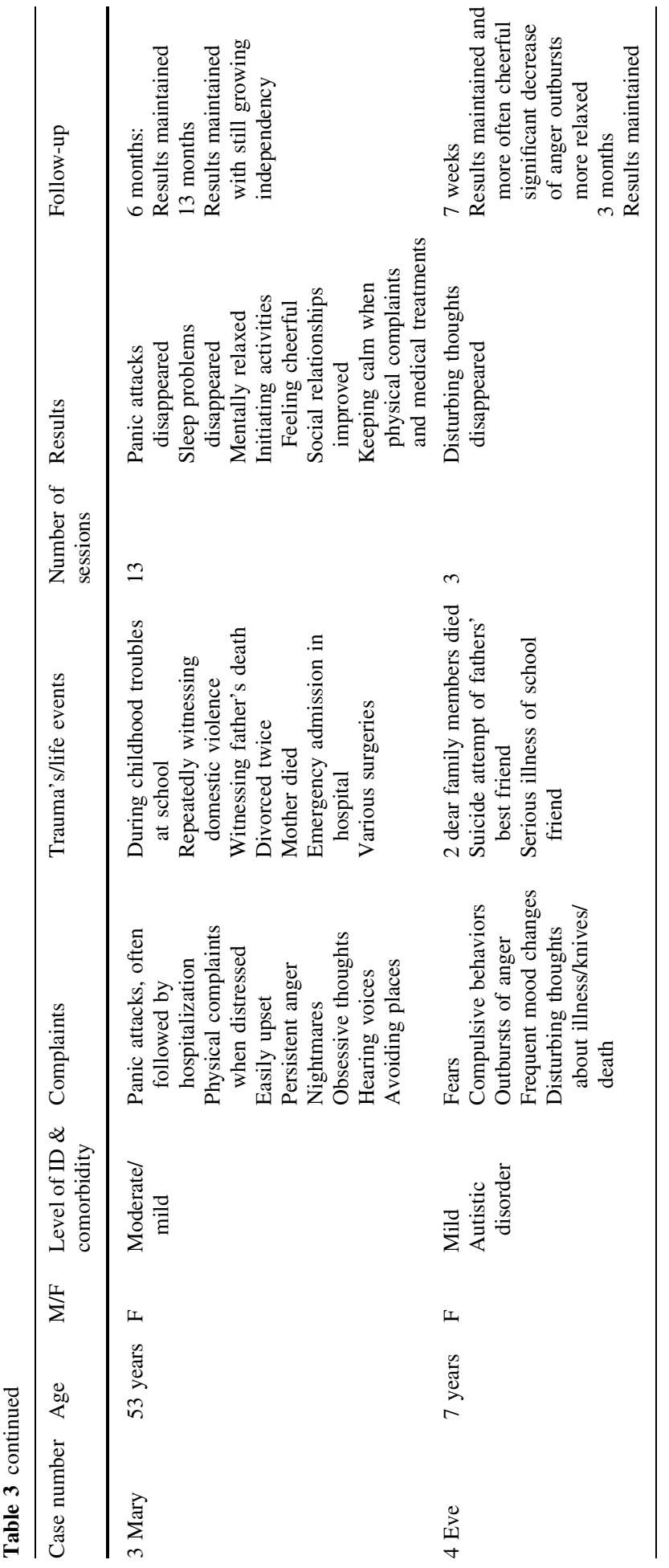


symptom, resulting in a diagnosis of multiple complex developmental disorder (MCDD; [27]). It should be noted that in subject \#2 treatment results maintained after phasing out anti-psychotic medication. Moreover, due to the mistake of diagnosing 'autism' in subject \#2 other PTSD symptoms seem to have been overshadowed. These assessment failures underscore the notion that until now the psychological impact of negative emotional events on people with ID has received little attention by professionals. This is particularly striking, given that people with ID seem to have a predisposition to the development of PTSD [5].

In conclusion, the results of the four single cases presented here show that PTSD symptoms can be identified with cooperation from parents or trusted caregivers. In all instances, PTSD symptoms decreased with results that were maintained at 3 months to 2.5 year follow-up. Moreover, depressive symptoms and physical complaints subsided and developmental growth increased, which included the improvement of social and adaptive skills. Given the length of the baseline period and the fact that treatment was limited to a maximum of 13 sessions, the improvements clearly suggest the applicability and potential effectiveness of EMDR in treating PTSD in clients with mild ID. Nevertheless, the application of evidence based treatment procedures for psychotrauma, such as EMDR and cognitive behavioral treatment (CBT), to the treatment of people with ID requires further and more rigorous investigation.

Acknowledgements We would like to express profound gratitude to Andrew Seubert and Francine Shapiro for their useful suggestions to improve the text of the manuscript.

Open Access This article is distributed under the terms of the Creative Commons Attribution Noncommercial License which permits any noncommercial use, distribution, and reproduction in any medium, provided the original author(s) and source are credited.

\section{References}

1. American Psychiatric Association: Diagnostic and Statistical Manual of Mental Disorders DSM-IV-TR, 4th edn. Washington DC, American Psychiatric Association, 2000.

2. Kessler RC, Chiu WT, Demler O, et al.: Prevalence, severity, and comorbidity of 12-month DSM IV disorders in the national comorbidity survey replication. Archives of General Psychiatry 62:617-627, 2005 .

3. Macklin ML, Litz LJ, McNally RJ, et al.: Lower precombat intelligence is a risk factor for posttraumatic stress disorder. Journal of Consulting and Clinical Psychology 2:323-326, 1998.

4. McNally RJ, Shin LM: Association of intelligence with severity of posttraumatic stress disorder symptoms in Vietnam combat veterans. The American Journal of Psychiatry 6:936-938, 1995.

5. Mevissen L, De Jongh A: PTSD and its treatment in people with intellectual disabilities. Clinical Psychology Review 30:308-316, 2010.

6. Fletcher R, Loschen E, Stavrakaki C, et al.: Diagnostic Manual-Intellectual Disability (DM-ID): A Textbook of Diagnosis of Mental Disorders in Persons with Intellectual Disability. Kingston, NY, NADD Press, 2007.

7. Cloitre M: Effective psychotherapies for posttraumatic stress disorder: a review and critique. CNS Spectrums. The International Journal of Neuropsychiatric Medicine 1 (suppl):32-43, 2009.

8. Bisson J, Ehlers A, Matthews R, et al.: Psychological treatments for chronic post-traumatic stress disorder. Systematic review and meta-analysis. British Journal of Psychiatry 190:97-104, 2007.

9. National Collaborating Centre for Mental Health: Post-Traumatic Stress Disorder: The Management of PTSD in Adults and Children in Primary and Secondary Care. London, National Institute for Clinical Excellence, 2005.

10. Rodenburg R, Benjamin A, De Roos C, et al.: Efficacy of EMDR in children: A meta-analyses. Clinical Psychology Review 29:599-606, 2009. 
11. Rodenburg R, Benjamin A, Meijer A M, et al.: Eye movement desensitization and reprocessing in an adolescent with epilepsy and mild intellectual disability. Epilepsy and Behavior 16:175-180, 2009.

12. Fernando K, Medlicott L: My shield will protect me against the ANTS: Treatment of PTSD in a client with an intellectual disability. Journal of Intellectual \& Developmental Disability 34:187-192, 2009.

13. Lemmon VA, Mizes JS: Effectiveness of exposure therapy: A study of posttraumatic stress disorder and mental retardation. Cognitive and Behavioural Practice 4:317-323, 2002.

14. Stenfert Kroese B, Thomas G: Treating chronic nightmares of sexual assault survivors with an intellectual disability-two descriptive case studies. Journal of Applied Research in Intellectual Disabilities 19:75-80, 2006.

15. Giltaij, H: Alsof er een stofzuiger door mijn hoofd is gegaan. EMDR bij mensen met een visuele en verstandelijke beperking, [As if a vacuum cleaner went through my head. EMDR in people with visual and intellectual disabilities]. Tijdschrift voor Kinder-\& Jeugdpsychotherapie 3:1-97, 2004.

16. Shapiro F: Special issue: EMDR overview: Theory, research, and areas of controversy. EMDR 12 Years after its introduction: Past and future research. Journal of Clinical Psychology 58:1-22, 2002.

17. Maxfield L: Current status and future directions for EMDR research. Journal of EMDR Practice and Research 1:6-14, 2007.

18. Shapiro F: Eye Movement Desensitization and Reprocessing: Basic Principles, Protocols and Procedures, 2nd edn. New York, Guilford Press, 2001.

19. De Jongh A, Ten Broeke E: Handboek EMDR: een geprotocolleerde behandelmethode voor de gevolgen van psychotrauma, 4th edn., 2009. Harcourt publishers, Amsterdam, 2003.

20. Tinker RH, Wilson SA: Through the Eyes of a Child. EMDR with Children. New York, Norton, 1999.

21. Lovett J: Small Wonders. Healing Childhood Trauma with EMDR. New York, The free press, 1999.

22. Greenwald R: Eye Movement Desensitization and Reprocessing (EMDR) in Child and Adolescent Psychotherapy. Northvale, Aronson, 1999.

23. De Roos C, Beer R, De Jongh A, et al.: EMDR-protocol voor kinderen tot 18 jaar. In: Ten Broeke E, De Jongh A, Oppenheim, H-J (Eds) Praktijkboek EMDR. Casusconceptualisatie en specifieke patiëntengroepen. Amsterdam, Harcourt, 2008.

24. Seubert, A: EMDR with Clients with Mental Disability. In: Shapiro R (Ed) EMDR Solutions, Pathways to Healing. New York, Norton, 2005.

25. Wechsler D: Manual for the Wechsler Intelligence Scale for Children-Revised. New York, The Psychological Corporation, 1974.

26. Haassen PP van, Steene G Vander, Bruyn EEJ et al.: Wechsler Intelligence Scale for Children-Revised. Nederlandstalige Uitgave, Lisse, Swets \& Zeitlinger, 1986.

27. Bruin E de, Nijs P de, Verheij F et al.: Multiple complex developmental disorder delineated from PDD-NOS. Journal of Autism and Developmental Disorders 37:1181-1191, 2007.

28. Kraijer DW, Kema GN: Sociale Redzaamheidsschaal-Z voor hoger niveau. SRZ-P. Handleiding. Vierde, uitgebreide uitgave. Amsterdam, Harcourt Assessment, 2004.

29. Noens I, Berckelaer-Onnes IA van: Making sense in a fragmentary world: Communication in people with autism and learning disability. Autism 8:197-218, 2004.

30. Mol S, Arntz SA, Metsemakers, JFM, et al.: Symptoms of post-traumatic stress disorder after nontraumatic events: Evidence from an open population study. British Journal of Psychiatry 186:494-499, 2005 .

\section{Author Biographies}

L. Mevissen, M.D. Clinical psychologist at Accare, Centre for Child and Adolescent Psychiatry, Deventer, The Netherlands.

R. Lievegoed, M.D. Psychiatrist at Zonnehuizen, Centre for Mental Health in Intellectual Disability, Zeist, The Netherlands.

A. de Jongh, Ph.D. Professor at the Department of Behavioural Sciences, Academic Centre for Dentistry Amsterdam (ACTA), Universiteit van Amsterdam and Vrije Universiteit, Amsterdam, The Netherlands. 